\title{
On a theorem of Cauchy-Kovalevskaya type for a class of nonlinear PDE's of higher order with deviating arguments
}

\author{
by Antoni Augustynowicz (Gdańsk)
}

\begin{abstract}
We prove an existence theorem of Cauchy-Kovalevskaya type for the equation

$$
D_{t} u(t, z)=f\left(t, z, u\left(\alpha^{(0)}(t, z)\right), D_{z} u\left(\alpha^{(1)}(t, z)\right), \ldots, D_{z}^{k} u\left(\alpha^{(k)}(t, z)\right)\right)
$$

where $f$ is a polynomial with respect to the last $k$ variables.

1. Introduction. We study the existence and uniqueness of solutions to the following Cauchy problem:

$$
\begin{aligned}
D_{t} u(t, z) & =f\left(t, z, u\left(\alpha^{(0)}(t, z)\right), D_{z} u\left(\alpha^{(1)}(t, z)\right), \ldots, D_{z}^{k} u\left(\alpha^{(k)}(t, z)\right)\right), \\
u(0, z) & =0 .
\end{aligned}
$$
\end{abstract}

The presence of deviating arguments $\alpha^{(1)}, \ldots, \alpha^{(k)}$ makes problem (1) difficult. The classical methods, such as the theory of characteristics, difference schemes for $k=1$, transformations to a differential-integral equation (when $k \geq 2$ and $f$ is linear with respect to the last variable), fail to work if $\alpha^{(k)}(t, z) \neq(t, z)$.

In the case of $k=1$ and real variables, applying the Banach contraction principle, the Neumann series and the Fourier series methods resulted in getting certain existence theorems for limited classes of deviating arguments (see [1]), and for some linear equations ([9], [5]). There are more effective methods concerning analytic solutions to (1). These methods are based on power series expansions ([2]-[4]), properties of the Bernstein classes of analytic functions ([11]) and on the Nagumo lemma $([6,7,10]$, [12]-[15]). The last method is used in the present paper.

1991 Mathematics Subject Classification: Primary 35A10; Secondary 35A07, 35G25, $35 \mathrm{R} 10$.

Key words and phrases: Cauchy-Kovalevskaya theorem, deviating argument, nonlinear equation, analytic solution. 
The classical Kovalevskaya counterexample $D_{t} u=D_{z}^{2} u, u(0, z)=$ $(1-z)^{-1}([8,12])$ shows that if $k>1$ then problem (1) may have no analytic solutions, even for elementary right-hand side. In $[6,7]$, existence results were obtained under the assumption that the deviating arguments are separated from the lateral boundary of the Haar pyramid. We relax this condition when the right-hand side in (1) is a polynomial with respect to the last $k$ variables.

2. Banach spaces $E_{p}$. Nagumo lemma. Let $\Omega$ be an open bounded subset of the complex plane $\mathbb{C}$ and

$$
\begin{gathered}
d(z)=\operatorname{dist}(z, \partial \Omega), \quad d(t, z)=d(z)-|t| / \eta, \\
G_{\eta}=\left\{(t, z) \in \mathbb{C}^{2}: z \in \Omega, d(t, z)>0,|t|<t_{0}\right\},
\end{gathered}
$$

where $\eta, t_{0}>0$ are fixed. The set $G_{\eta}$ is the Haar pyramid with slope $\eta$, and $d(t, z)$ is the distance between $(t, z)$ and the boundary of $t$-intersection of $G_{\eta}$.

Let $H(G)$ denote the space of all analytic functions on $G$. For $p \geq 0$ and $u \in H\left(G_{\eta}\right)$ we define

$$
\|u\|_{p}=\sup _{(t, z) \in G_{\eta}}|u(t, z)| d(t, z)^{p}, \quad E_{p}=\left\{u \in H\left(G_{\eta}\right):\|u\|_{p}<+\infty\right\} .
$$

The set $E_{p}$ is a Banach space with the natural linear structure and the norm $\|\cdot\|_{p}$.

Our investigations are based on the following

LEMma 1. If $a, u \in H\left(G_{\eta}\right)$, then

(1) $\left\|D_{z} u\right\|_{p+1} \leq C_{p}\|u\|_{p}$, where $C_{p}=(p+1)(1+1 / p)^{p}, C_{0}=1$,

(2) $\|a(\cdot) u(\cdot)\|_{p+q} \leq\|a\|_{q}\|u\|_{p}$,

(3) $\|u(\alpha(\cdot))\|_{p} \leq \lambda_{\alpha}^{p}\|u\|_{p}$ if $\alpha\left(G_{\eta}\right) \subset G_{\eta}$, where

$$
\lambda_{\alpha}=\sup _{(t, z) \in G_{\eta}} \frac{d(t, z)}{d(\alpha(t, z))},
$$

(4) $\|I u\|_{p} \leq(\eta / p)\|u\|_{p+1}$, where $(I u)(t, z)=\int_{0}^{t} u(s, z) d s$.

The assertion (1) is the Nagumo lemma (cf. [10]). Conditions (2)-(3), (4) are proved in [6], [13], respectively.

3. Existence and uniqueness results. In order to present the main idea, we consider a simple case of equation (1):

$$
\begin{aligned}
D_{t} u(t, z) & =a(t, z, u(\alpha(t, z)))\left(D_{z}^{k} u(\beta(t, z))\right)^{n}+b(t, z, u(\gamma(t, z))), \\
u(0, z) & =0 .
\end{aligned}
$$


TheOREM 1. Suppose that for some $r, h>0$ and $\kappa \in(0,1)$, there exist $\omega \in[0, \kappa), \lambda, \eta>0$, and $A, B \geq 0$ such that $a, b$ are analytic on $G_{\eta} \times \bar{K}(0, r)$ (where $\bar{K}(0, r)$ is the closed ball in $\mathbb{C}$ centered at the origin and with radius $r)$, and $\alpha, \beta, \gamma: G_{\eta} \rightarrow G_{\eta}$ are analytic. Assume that for $(t, z) \in G_{\eta}$, $|u| \leq r$, we have

$$
\begin{gathered}
\|a(\cdot, u)\|_{\omega} \leq A, \quad\|b(\cdot, u)\|_{\kappa} \leq B, \\
d(t, z)^{\kappa-\omega} \leq \lambda d(\beta(t, z))^{n(\kappa+k-1)}, \\
\frac{\eta}{1-\kappa} \widehat{d}^{1-\kappa}\left[A \lambda\left(C_{\kappa, k-1} h\right)^{n}+B\right] \leq r, \\
\frac{\eta}{\kappa}\left[A \lambda\left(C_{\kappa, k-1} h\right)^{n}\left(C_{\omega}+C_{\kappa-\omega}\right)+C_{\kappa} B\right] \leq h,
\end{gathered}
$$

where

$$
\widehat{d}=\sup _{(t, z) \in G_{\eta}} d(t, z), \quad C_{p, j}=C_{p} C_{p+1} \ldots C_{p+j-1}, \quad C_{p, 0}=1 .
$$

Then problem (2) has an analytic solution defined on $G_{\eta}$. Moreover, if there exist constants $\lambda_{1}, \lambda_{3}, p>0$ and $A^{\prime}, B^{\prime}, \omega^{\prime}, \kappa^{\prime} \geq 0$ such that

$$
\begin{gathered}
|a(t, z, u)-a(t, z, v)| \leq A^{\prime} d(t, z)^{-\omega^{\prime}}|u-v|, \\
|b(t, z, u)-b(t, z, v)| \leq B^{\prime} d(t, z)^{-\kappa^{\prime}}|u-v|, \\
d(t, z)^{p+1+\omega-\kappa-\omega^{\prime}} \leq \lambda_{1} d(\alpha(t, z))^{p}, \quad d(t, z)^{p+1-\kappa^{\prime}} \leq \lambda_{3} d(\gamma(t, z))^{p}, \\
L=\frac{\eta}{p}\left[A^{\prime}\left(C_{\kappa, k-1} h\right)^{n} \lambda \lambda_{1}+A n\left(C_{\kappa, k-1} h\right)^{n-1} C_{p, k} \lambda \lambda_{2}^{p+1-\kappa}+B^{\prime} \lambda_{3}\right]<1
\end{gathered}
$$

for $(t, z) \in G_{\eta},|u|,|v| \leq r$, where $\lambda_{2}=\sup \left\{d(t, z) d(\beta(t, z))^{-1}:(t, z) \in G_{\eta}\right\}$, then the solution is unique in the set

$$
D=\left\{u \in E_{0}:\|u\|_{0} \leq r,\left\|D_{z} u\right\|_{\kappa} \leq h\right\} .
$$

REMARK 1. If $\delta: G_{\eta} \rightarrow G_{\eta}$ and $d(t, z) \leq \tau d(\delta(t, z))$, then $d(t, z)^{q} \leq$ $\tau^{\prime} d(\delta(t, z))^{q^{\prime}}$ for $q \geq q^{\prime}$ and some $\tau^{\prime}>0$. This shows that the existence of constants $\lambda_{1}$ and $\lambda_{3}$ follows from the natural assumption

$d(t, z) \leq \tau_{1} d(\alpha(t, z)), \quad d(t, z) \leq \tau_{2} d(\gamma(t, z)) \quad$ if $\kappa+\omega^{\prime} \leq 1+\omega$ and $\kappa^{\prime} \leq 1$.

Since $\kappa-\omega \leq n(\kappa+k-1)$, from (3) we have $\lambda_{2}<+\infty$. Observe also that $L<1$ and the last two inequalities in (3) are satisfied, provided $\eta$ is sufficiently small.

Proof (of Theorem 1). Define

$$
(F u)(t, z)=\int_{0}^{t}\left[a(s, z, u(\alpha(s, z)))\left(D_{z}^{k} u(\beta(s, z))\right)^{n}+b(s, z, u(\gamma(s, z)))\right] d s .
$$


We now prove that $F(D) \subset D$. If $u \in D$ then

$$
\begin{aligned}
\left|\left(D_{z}^{k} u(\beta(t, z))\right)^{n}\right| & \leq\left(\left\|D_{z}^{k} u\right\|_{\kappa+k-1} d(\beta(t, z))^{-\kappa-k+1}\right)^{n} \\
& \leq\left(C_{\kappa, k-1}\left\|D_{z} u\right\|_{\kappa}\right)^{n} d(\beta(t, z))^{-n(\kappa+k-1)} \\
& \leq\left(C_{\kappa, k-1} h\right)^{n} \lambda d(t, z)^{-\kappa+\omega}
\end{aligned}
$$

so we obtain

$$
\begin{aligned}
\left|D_{t}(F u)(t, z)\right| & \leq A \lambda d(t, z)^{-\omega} d(t, z)^{-\kappa+\omega}\left(C_{\kappa, k-1} h\right)^{n}+B d(t, z)^{-\kappa} \\
& =\left(A \lambda\left(C_{\kappa, k-1} h\right)^{n}+B\right) d(t, z)^{-\kappa}
\end{aligned}
$$

hence

$$
|(F u)(t, z)| \leq \frac{\eta}{1-\kappa} \widehat{d}^{1-\kappa}\left[A \lambda\left(C_{\kappa, k-1} h\right)^{n}+B\right] \leq r .
$$

Moreover, we get

$$
\begin{aligned}
\left|D_{t} D_{z}(F u)(t, z)\right| \leq & A C_{\omega} d(t, z)^{-\omega-1}\left(C_{\kappa, k-1} h d(\beta(t, z))^{-\kappa-k+1}\right)^{n} \\
& +A d(t, z)^{-\omega}\left|\frac{\partial}{\partial z}\left(D_{z}^{k} u(\beta(t, z))\right)^{n}\right|+C_{\kappa} B d(t, z)^{-\kappa-1} \\
\leq & A C_{\omega}\left(C_{\kappa, k-1} h\right)^{n} \lambda d(t, z)^{-\kappa-1} \\
& +A \lambda C_{\kappa-\omega}\left(C_{\kappa, k-1} h\right)^{n} d(t, z)^{-\kappa-1}+C_{\kappa} B d(t, z)^{-\kappa-1}
\end{aligned}
$$

hence

$$
\left|D_{z}(F u)(t, z)\right| d(t, z)^{\kappa} \leq \frac{\eta}{\kappa}\left[A \lambda\left(C_{\kappa, k-1} h\right)^{n}\left(C_{\omega}+C_{\kappa-\omega}\right)+C_{\kappa} B\right] \leq h
$$

and $F u \in D$. The set $D$ is a convex and compact subset of $E_{q}$ for every $q>0$. We now prove that the operator $F$ is continuous on $D$ with respect to the norm $\|\cdot\|_{q}$, provided $q$ is sufficiently large. For any $u, v \in D$, we have

$$
\begin{aligned}
& |(F u)(t, z)-(F v)(t, z)| \\
& \leq \int_{0}^{|t|}|a(s, z, u(\alpha(s, z)))-a(s, z, v(\alpha(s, z)))|\left|D_{z}^{k} u(\beta(s, z))\right|^{n}|d s| \\
& \quad+\int_{0}^{|t|}|a(s, z, v(\alpha(s, z)))|\left|\left(D_{z}^{k} u(\beta(s, z))\right)^{n}-\left(D_{z}^{k} v(\beta(s, z))\right)^{n}\right||d s| \\
& \quad+\int_{0}^{|t|}|b(s, z, u(\gamma(s, z)))-b(s, z, v(\gamma(s, z)))||d s| \\
& \leq \int_{0}^{|t|}|a(s, z, u(\alpha(s, z)))-a(s, z, v(\alpha(s, z)))| \\
& \quad \times\left(C_{q, k-1}\left\|D_{z} u\right\|_{q} d(\beta(s, z))^{-q-k+1}\right)^{n}|d s|
\end{aligned}
$$




$$
\begin{aligned}
& +\int_{0}^{|t|} A d(s, z)^{-\omega} n\left[C_{q, k-1} \max \left\{\left\|D_{z} u\right\|_{q},\left\|D_{z} v\right\|_{q}\right\} d(\beta(s, z))^{-q-k+1}\right]^{n-1} \\
& \times\left|D_{z}^{k} u(\beta(s, z))-D_{z}^{k} v(\beta(s, z))\right||d s| \\
& +\int_{0}^{|t|}|b(s, z, u(\gamma(s, z)))-b(s, z, v(\gamma(s, z)))||d s| \\
& \leq\left(C_{q, k-1}\left\|D_{z} u\right\|_{q}\right)^{n} \sup _{\mu \in[0,1]}|a(\mu t, z, u(\alpha(\mu t, z)))-a(\mu t, z, v(\alpha(\mu t, z)))| \\
& \times d(\mu t, z) \int_{0}^{|t|} d(s, z)^{-q-1} \frac{d(s, z)^{q}}{d(\beta(s, z))^{n(q+k-1)}}|d s| \\
& +A n\left[C_{q, k-1} \max \left\{\left\|D_{z} u\right\|_{q},\left\|D_{z} v\right\|_{q}\right\}\right]^{n-1} \\
& \times \int_{0}^{|t|} d(s, z)^{-\omega} d(\beta(s, z))^{-(n-1)(q+k-1)} C_{q, k}\|u-v\|_{q} d(\beta(s, z))^{-q-k}|d s| \\
& +\int_{0}^{|t|}|b(s, z, u(\gamma(s, z)))-b(s, z, v(\gamma(s, z)))||d s| .
\end{aligned}
$$

Since

$$
\begin{aligned}
\sup _{(s, z) \in G_{\eta}} \frac{d(s, z)^{q}}{d(\beta(s, z))^{n q+m}} & \\
& \leq \lambda^{q /(\kappa-\omega)} \sup _{(s, z) \in G_{\eta}} \frac{d(\beta(s, z))^{q n(\kappa+k-1) /(\kappa-\omega)}}{d(\beta(s, z))^{n q+m}} \\
& =\lambda^{q /(\kappa-\omega)} \sup _{(s, z) \in G_{\eta}} d(\beta(s, z))^{q n(k-1+\omega) /(\kappa-\omega)-m}<+\infty
\end{aligned}
$$

for any $m>0$ and for sufficiently large $q$, there exists a constant $c$ such that

$$
\|F u-F v\|_{q} \leq c\|u-v\|_{q}+c \sup _{(t, z) \in G_{\eta}} \Delta_{u, v}(t, z) d(t, z)
$$

for some $q>0$, where

$$
\begin{aligned}
\Delta_{u, v}(s, z)= & |a(s, z, u(\alpha(s, z)))-a(s, z, v(\alpha(s, z)))| \\
& +|b(s, z, u(\gamma(s, z)))-b(s, z, v(\gamma(s, z)))| .
\end{aligned}
$$

Fix $u \in D$. Let $d_{0}>0$ and $G\left(d_{0}\right)=\left\{(t, z) \in G_{\eta}: d(t, z) \geq d_{0}\right\}$. Then we get

$$
\begin{aligned}
\|F u-F v\|_{q} \leq & c\|u-v\|_{q}+c \sup _{(t, z) \in G_{\eta} \backslash G\left(d_{0}\right)} \Delta_{u, v}(t, z) d(t, z) \\
& +c \sup _{(t, z) \in G\left(d_{0}\right)} \Delta_{u, v}(t, z) d(t, z)=S_{1}+S_{2}+S_{3} .
\end{aligned}
$$


We prove that $S_{1}+S_{2}+S_{3}$ tends to zero if $v$ tends to $u$ in the norm $\|\cdot\|_{q}$. Since $u, v \in D$, we have

$$
\Delta_{u, v}(t, z) d(t, z) \leq 2 A d(t, z)^{1-\omega}+2 B d(t, z)^{1-\kappa},
$$

hence $S_{2}$ becomes small when $d_{0}$ is small enough. Given any fixed $d_{0}$, we observe that the functions $a, b$ are uniformly continuous on $G\left(d_{0}\right) \times \bar{K}(0, r)$ and the functions $\alpha, \gamma$ are uniformly continuous on $G\left(d_{0}\right)$. Therefore, $S_{3} \rightarrow 0$ as $\|v-u\|_{q} \rightarrow 0$. This proves the continuity of $F$ on $D$. The Schauder fixed point theorem completes the proof of the first assertion.

Applying conditions (4) with $u, v \in D,(t, z) \in G_{\eta}$, we have

$$
\begin{aligned}
\mid D_{t}[( & F u)-(F v)](t, z) \mid \\
\leq & |a(t, z, u(\alpha(t, z)))-a(t, z, v(\alpha(t, z)))|\left|D_{z}^{k} u(\beta(t, z))\right|^{n} \\
& +|a(t, z, v(\alpha(t, z)))|\left|\left(D_{z}^{k} u(\beta(t, z))\right)^{n}-\left(D_{z}^{k} v(\beta(t, z))\right)^{n}\right| \\
& +|b(t, z, u(\gamma(t, z)))-b(t, z, v(\gamma(t, z)))| \\
\leq & A^{\prime} d(t, z)^{-\omega^{\prime}}|u(\alpha(t, z))-v(\alpha(t, z))| d(\alpha(t, z))^{p} d(\alpha(t, z))^{-p} \\
& \times\left(C_{\kappa, k-1} h d(\beta(t, z))^{-\kappa-k+1}\right)^{n} \\
& +A d(t, z)^{-\omega} n\left(C_{\kappa, k-1} h d(\beta(t, z))^{-\kappa-k+1}\right)^{n-1} \\
& \times\left|D_{z}^{k} u(\beta(t, z))-D_{z}^{k} v(\beta(t, z))\right| \\
& +B^{\prime} d(t, z)^{-\kappa^{\prime}}|u(\gamma(t, z))-v(\gamma(t, z))| d(\gamma(t, z))^{p} d(\gamma(t, z))^{-p} \\
\leq & A^{\prime}\left(C_{\kappa, k-1} h\right)^{n}\|u-v\|_{p} d(t, z)^{-\omega^{\prime}} d(\alpha(t, z))^{-p} d(\beta(t, z))^{-n(\kappa+k-1)} \\
& +A n\left(C_{\kappa, k-1} h\right)^{n-1} C_{p, k}\|u-v\|_{p} \\
& \times d(t, z)^{-\omega} d(\beta(t, z))^{-(n-1)(\kappa+k-1)} d(\beta(t, z))^{-k-p} \\
& +B^{\prime}\|u-v\|_{p} d(t, z)^{-\kappa^{\prime}} d(\gamma(t, z))^{-p} \\
\leq & {\left[A^{\prime}\left(C_{\kappa, k-1} h\right)^{n} \lambda \lambda_{1}+A n\left(C_{\kappa, k-1} h\right)^{n-1} C_{p, k} \lambda \lambda_{2}^{p+1-\kappa}+B^{\prime} \lambda_{3}\right] } \\
& \times\|u-v\|_{p} d(t, z)^{-p-1},
\end{aligned}
$$

hence $\|F u-F v\|_{p} \leq L\|u-v\|_{p}$ and $F$ is contractive on $D$ with respect to the norm $\|\cdot\|_{p}$. The Banach contraction principle completes the proof.

REMARK 2. Theorem 1 only gives a local existence (and uniqueness) result. Assume that $\left|\alpha_{0}(t, z)\right|,\left|\beta_{0}(t, z)\right|,\left|\gamma_{0}(t, z)\right|<|t|$ for $0<|t|<T\left(\alpha_{0}\right.$, $\beta_{0}, \gamma_{0}$ are the time-coordinates of $\alpha, \beta, \gamma$ respectively), and $a, b$ are analytic on $\Omega \times K(0, T) \times \mathbb{C}$. Then we can extend any local solution of $(2)$ to the set $\Omega \times K(0, T)$ by a step-by-step method. Assumption (3) of Theorem 1 is 
essential, and it is satisfied when there exists $d_{0}>0$ such that $d(\beta(t, z)) \geq d_{0}$ for $(t, z) \in G_{\eta}$. Such a condition is assumed in [6], [7]. One may expect that (3) cannot be satisfied when

$$
\inf \left\{d(\beta(t, z)):(t, z) \in G_{\eta}\right\}=0 .
$$

We demonstrate in the Example below that, taking any $k, n, \kappa, \omega$, there exists a deviating argument $\beta$ which is not separated from the lateral boundary of the Haar pyramid, but (3) is satisfied. Moreover, the assumptions of Theorem 1 require $\eta$ to be small enough. The deviating argument in the Example transforms $G_{\eta}$ into itself for any $\eta, t_{0}>0$ sufficiently small.

ExAmple. Take $r \geq 2^{m /(m-1)}, m>1$. Define

$$
\Omega=\{z \in \mathbb{C}:|z|<r,|\arg z|<\pi / 2\} .
$$

Take further

$$
a \in \mathbb{C}, \quad a \neq 0, \quad 0<\eta<\frac{2 m-1}{m^{2}|a|}, \quad 0<t_{0} \leq \frac{1}{|a| b}, \quad b=r^{(m-1) / m} .
$$

We have

$$
d(z)=\min \{\operatorname{Re} z, r-|z|\} .
$$

Define

$$
\beta(t, z)=\left(a t^{2}, z^{1 / m}\right), \quad\left|\arg z^{1 / m}\right|<\frac{\pi}{2 m} .
$$

We prove that $\beta\left(G_{\eta}\right) \subset G_{\eta}$. Since $r>1$, it is easily seen that $\beta(0, z) \in \Omega$ if $z \in \Omega$. Let

$$
z^{1 / m}=x \exp (i \phi), \quad x \in\left(0, r^{1 / m}\right), \quad|\phi|<\frac{\pi}{2 m} .
$$

We get $r \geq 2 r^{1 / m} \geq x(1+\cos \phi)$, so $r-\left|z^{1 / m}\right|=r-x \geq x \cos \phi=\operatorname{Re} z^{1 / m}$, hence

$$
d\left(z^{1 / m}\right)=\operatorname{Re} z^{1 / m}, \quad z \in \Omega,
$$

and

$$
\frac{d(z)}{d\left(z^{1 / m}\right)^{m}} \leq \frac{\operatorname{Re} z}{\left(\operatorname{Re} z^{1 / m}\right)^{m}}=\frac{x^{m} \cos m \phi}{(x \cos \phi)^{m}}=\frac{\cos m \phi}{\cos ^{m} \phi} \leq 1 .
$$

In particular,

$$
\begin{gathered}
d(z) \leq \sup _{y \in \Omega}\left(d\left(y^{1 / m}\right)\right)^{m-1} d\left(z^{1 / m}\right) \leq b d\left(z^{1 / m}\right), \\
d(\beta(t, z))=d\left(z^{1 / m}\right)-\frac{\left|a t^{2}\right|}{\eta} \geq \frac{1}{b} d(z)-\frac{1}{b} \frac{|t|}{\eta}=\frac{d(t, z)}{b}>0,
\end{gathered}
$$

if $(t, z) \in G_{\eta}$. This implies $\beta\left(G_{\eta}\right) \subset G_{\eta}$. Now we prove that

$$
A(t, z)=\frac{d(t, z)}{d(\beta(t, z))^{m}} \leq 1 .
$$


We have

$$
A(t, z) \leq \frac{\operatorname{Re} z-|t| / \eta}{\left(\operatorname{Re} z^{1 / m}-\left|a t^{2}\right| / \eta\right)^{m}} .
$$

The estimate $\eta<(2 m-1) /\left(m^{2}|a|\right)$ and the inequality $\cos m \phi \leq \cos \phi \leq 1$ imply that the right-hand side of the above inequality is decreasing in $|t| \in$ $[0, \eta \operatorname{Re} z)$, thus its maximum is reached at $|t|=0$, hence

$$
A(t, z) \leq \frac{\operatorname{Re} z}{\left(\operatorname{Re} z^{1 / m}\right)^{m}} \leq 1
$$

Estimate (5) is optimal. Indeed, $A(0, z)=1$ if $\operatorname{Im} z=0$ and $\operatorname{Re} z<1$. It follows from (5) that, if $m(\kappa-\omega) \geq n(\kappa+k-1)$, then

$$
d(t, z)^{\kappa-\omega} \leq d(\beta(t, z))^{m(\kappa-\omega)} \leq \widehat{d}^{m(\kappa-\omega)-n(\kappa+k-1)} d(\beta(t, z))^{n(\kappa+k-1)},
$$

therefore (3) is satisfied.

We generalize Theorem 1 to the equation

$$
\begin{aligned}
D_{t} u(t, z)= & \sum_{n=1}^{N} \sum_{\left|k_{n}\right| \leq K} a_{k_{n}}\left(t, z, u\left(\alpha_{k_{n}}(t, z)\right)\right) \prod_{i=1}^{n} D_{z}^{k_{n i}} u\left(\beta_{k_{n}, i}(t, z)\right) \\
& +b(t, z, u(\gamma(t, z)))
\end{aligned}
$$

where $k_{n}=\left(k_{n 1}, \ldots, k_{n n}\right)$ is such that $k_{n i} \geq 1$ and $\left|k_{n}\right|=k_{n 1}+\ldots+k_{n n}$.

If all coefficients $a_{k_{n}}$ vanish but one $\left(k_{n_{0}}=(k, \ldots, k)\right)$ and $\beta_{k_{n}, i}=\beta, i=$ $1, \ldots, n$, then the above equation becomes equation (2).

TheOREM 2. Suppose that there are $r, h>0, \kappa \in(0,1)$, and $\omega_{k_{n}} \in$ $[0, \kappa), \eta, \lambda_{k_{n}}>0, A_{k_{n}}, B \geq 0$ such that $a_{k_{n}}, b$ are analytic functions on $G_{\eta} \times \bar{K}(0, r)$, and the functions $\alpha_{k_{n}}, \beta_{k_{n}, i}, \gamma$ map $G_{\eta}$ into itself. Assume that, for $(t, z) \in G_{\eta},|u| \leq r$, we have

$$
\begin{gathered}
\left\|a_{k_{n}}(\cdot, u)\right\|_{\omega_{k_{n}}} \leq A_{k_{n}}, \quad\|b(\cdot, u)\|_{\kappa} \leq B, \\
d(t, z)^{\kappa-\omega_{k_{n}}} \leq \lambda_{k_{n}} \prod_{i=1}^{n} d\left(\beta_{k_{n}, i}(t, z)\right)^{\kappa+k_{n i}-1}, \\
\frac{\eta}{1-\kappa} \widehat{d}^{1-\kappa}\left[B+\sum_{n=1}^{N} \sum_{\left|k_{n}\right| \leq K} A_{k_{n}} h^{n} \prod_{i=1}^{n} C_{\kappa, k_{n i}-1}\right] \leq r, \\
\frac{\eta}{\kappa}\left[C_{\kappa} B+\sum_{n=1}^{N} \sum_{\left|k_{n}\right| \leq K} A_{k_{n}} h^{n} \lambda_{k_{n}}\left(C_{\omega_{k_{n}}}+C_{\kappa-\omega_{k_{n}}}\right) \prod_{i=1}^{n} C_{\kappa, k_{n i}-1}\right] \leq h .
\end{gathered}
$$

Then there exists an analytic solution to the homogeneous Cauchy problem for equation (6) in the set D. Moreover, if there exist constants $p, \lambda_{k_{n}}^{(1)}, \lambda^{(3)}$ $>0, A_{k_{n}}^{\prime}, B^{\prime}, \omega_{k_{n}}^{\prime}, \kappa^{\prime} \geq 0$ such that

$$
\left|a_{k_{n}}(t, z, u)-a_{k_{n}}(t, z, v)\right| \leq A_{k_{n}}^{\prime} d(t, z)^{-\omega_{k_{n}}^{\prime}}|u-v|,
$$




$$
\begin{aligned}
& |b(t, z, u)-b(t, z, v)| \leq B^{\prime} d(t, z)^{-\kappa^{\prime}}|u-v|, \\
& d(t, z)^{p+1-\kappa+\omega_{k_{n}}-\omega_{k_{n}}^{\prime}} \leq \lambda_{k_{n}}^{(1)} d\left(\alpha_{k_{n}}(t, z)\right)^{p}, \quad d(t, z)^{p+1-\kappa^{\prime}} \leq \lambda^{(3)} d(\gamma(t, z))^{p}, \\
& \frac{\eta}{p}\left\{\sum _ { n = 1 } ^ { N } \sum _ { | k _ { n } | \leq K } \lambda _ { k _ { n } } \left[A_{k_{n}}^{\prime} h^{n}\left(\prod_{i=1}^{n} C_{\kappa, k_{n i}-1}\right) \lambda_{k_{n}}^{(1)}\right.\right. \\
& \left.\left.+A_{k_{n}} h^{n-1} \sum_{j=1}^{n}\left(\prod_{i=1, i \neq j}^{n} C_{\kappa, k_{n i}-1}\right) C_{p, k_{n j}} \lambda_{k_{n}, j}^{(2)}\right]+B^{\prime} \lambda^{(3)}\right\}<1,
\end{aligned}
$$

for $(t, z) \in G_{\eta},|u|,|v| \leq r$, where

$$
\lambda_{k_{n}, j}^{(2)}=\sup _{(t, z) \in G_{\eta}}\left(\frac{d(t, z)}{d\left(\beta_{k_{n}, j}(t, z)\right)}\right)^{p+1-\kappa},
$$

then the solution is unique in $D$.

We omit the proof, because its idea is similar to that of the proof of Theorem 1.

The results of this paper can be easily generalized for a multidimensional variable $z$ and a strongly coupled system of equations. Moreover, the results hold true in the real case, i.e. for functions $u$ of variables $(t, z) \in G_{\eta} \subset$ $\mathbb{R} \times \mathbb{C}$ of class $C^{1}$ in $t$ and analytic in $z$. It suffices to assume that the first coordinates of the deviating arguments of the unknown function are independent of $z$.

\section{References}

[1] A. Augustynowicz, Existence and uniqueness of solutions for partial differentialfunctional equations of the first order with deviating arguments of the derivative of unknown function, Serdica Math. J. 23 (1997), 203-210.

[2] - Analytic solutions to the first order partial differential equations with time delays at the derivatives, Funct. Differ. Equations 6 (1999), 19-29.

[3] A. Augustynowicz and H. Leszczyński, On the existence of analytic solutions of the Cauchy problem for first-order partial differential equations with retarded variables, Comment. Math. Prace Mat. 36 (1996), 11-25.

[4] - - - On x-analytic solutions to the Cauchy problem for partial differential equations with retarded variables, Z. Anal. Anwendungen 15 (1996), 345-356.

$[5-,-$, Periodic solutions to the Cauchy problem for PDEs with retarded variables, submitted.

[6] A. Augustynowicz, H. Leszczyński and W. Walter, Cauchy-Kovalevskaya theory for equations with deviating variables, Aequationes Math. 58 (1999), 143156.

[7] - - - - Cauchy-Kovalevskaya theory for nonlinear equations with deviating variables, Nonlinear Anal., to appear.

[8] S. von Kowalevsky, Zur Theorie der partiellen Differentialgleichungen, J. Reine Angew. Math. 80 (1875), 1-32. 
[9] H. Leszczyński, Fundamental solutions to linear first-order equations with a delay at derivatives, Boll. Un. Mat. Ital. A (7) 10 (1996), 363-375.

[10] M. Nagumo, Über das Anfangswertproblem partieller Differentialgleichungen, Japan. J. Math. 18 (1942), 41-47.

[11] R. M. Redheffer and W. Walter, Existence theorems for strongly coupled systems of partial differential equations over Bernstein classes, Bull. Amer. Math. Soc. 82 (1976), 899-902.

[12] W. Walter, An elementary proof of the Cauchy-Kovalevsky Theorem, Amer. Math. Monthly 92 (1985), 115-126.

[13] - Functional differential equations of the Cauchy-Kovalevsky type, Aequationes Math. 28 (1985), 102-113.

[14] T. Yamanaka, A Cauchy-Kovalevskaja type theorem in the Gevrey class with a vector-valued time variable, Comm. Partial Differential Equations 17 (1992), 14571502 .

[15] T. Yamanaka and H. Tamaki, Cauchy-Kovalevskaya theorem for functional partial differential equations, Comment. Math. Univ. St. Paul. 29 (1980), 55-64.

Institute of Mathematics

University of Gdańsk

Wita Stwosza 57

80-952 Gdańsk, Poland

E-mail: antek@ksinet.univ.gda.pl

Reçu par la Rédaction le 30.11.1998

Révisé le 30.4.1999 